\title{
A Time-Motion Study on the Operating Room Processes among Pregnant COVID-19 Patients Undergoing Cesarean Section in a Tertiary Government Hospital
}

\author{
Ma. Evita D. dela Cruz-Tabanda, MD and Maria Angela R. Bandola, MD \\ Division of Infectious Diseases. Department of Obstetrics and Gynecology, Philippine General Hospital, University of the Philippines Manila
}

\begin{abstract}
Objective. This study aims to determine time and motion in the operating room in emergent, urgent and scheduled cesarean section surgeries among pregnant COVID-19 patients.
\end{abstract}

Methodology. A time and motion performance evaluation study was done by computing the following parameters: pre-induction time, pre-incision time, opening time, closing time, for both decision-to-delivery interval (DDI) and overall operative time.

Results. During the study period, emergent DDI average was 2 hours and 38 minutes, emergent overall operative time was 1 hour and 31minutes, urgent DDI average was 3 hours and 51 minutes, and urgent overall operative time of 1 hour and 57 minutes. However, in both urgent and emergent cases, the recommended DDI of 30 minutes, and the average duration of 44.3 minutes for CS were not feasible.

Conclusion. The COVID-19 pandemic has negatively affected the provision of surgical obstetric care and OR utilization. Due to the new safety protocol for healthcare workers and patients, there was a significant delay in DDI and overall operative time. The causes were preparation, anesthesia factors or obstetrician factors. Identifying modifiable obstacles may improve the DDI, overall operative time, and the quality of maternal and child birth care during this pandemic.

Key Words: cesarean section, COVID-19, decision-to-delivery Interval (DDI), time and motion study

\section{INTRODUCTION}

Corresponding author: Ma. Evita D. dela Cruz-Tabanda, MD Division of Infectious Diseases

Department of Obstetrics and Gynecology

Philippine General Hospital

University of the Philippines Manila

Taft Avenue, Ermita, Manila 1000, Philippines

Email: mddelacruz19@up.edu.ph
The novel coronavirus or SARS CoV-2 was first isolated in Wuhan City, Hubei province, in China last December 2019. Cases spread in several countries, and last March 11, 2020, the World Health Organization (WHO) declared COVID-19 a pandemic. As of August 31, 2020, a total of 25,254,339 confirmed cases with 846,985 confirmed deaths were reported worldwide. ${ }^{1}$ According to the Department of Health (DOH) in the Philippines, the first case was identified last January 30, 2020 and as of August 31, 2020 a total of 220,819 confirmed cases with 3,558 confirmed deaths were reported. ${ }^{2}$ Due to the extensive morbidity and non-selective and persistent spread, it was possible to see women with COVID-19 in all trimesters of pregnancy.

Coronavirus is a single-stranded, non-segmented, enveloped RNA virus. It can be transmitted primarily from person to person via droplets released from the respiratory 
tract of an individual directly onto a mucosal surface or conjunctivae of another or through contact via direct or indirectly from contaminated fomites. ${ }^{1}$ Common symptoms are fever, cough, and myalgia or fatigue. Less common symptoms are sputum production, headache, hemoptysis and diarrhea. ${ }^{3}$ The clinical presentation of SARS CoV-2 ranges from mild common cold-like illness to severe viral pneumonia, which can cause acute respiratory distress syndrome that is potentially fatal. ${ }^{4}$

The Philippine Infectious Diseases Society for Obstetrics and Gynecology (PIDSOG) released a handbook for obstetrician-gynecologists and other healthcare providers in delivering the most ideal and applicable management to the Filipino gravidas and their unborn children. It recommends that all pregnant patients should be triaged and screened for symptoms such as fever, cough, difficulty of breathing or shortness of breath, and gastrointestinal symptoms. Unless proven otherwise, all health care providers should follow the Centers for Disease Control and prevention $(\mathrm{CDC})$ personal protective equipment (PPE), i.e., to wear a surgical mask, protective eyewear, gown, gloves, and N-95 for confirmed or suspected COVID-19 patients. A designated isolation room should be reserved for these patients and health care workers should observe hand hygiene after every patient contact and proper donning and doffing of PPE. ${ }^{5}$

The route of delivery should be based on standard obstetric indications and clinical urgency. Vaginal delivery is not contraindicated. In critically-ill parturient- rapid maternal deterioration, difficulty in mechanical ventilation due to gravid uterus and fetal compromise, emergent delivery is recommended. Delivery, either vaginally or abdominally, should be performed with respiratory precautions wearing complete PPE and in rooms with negative pressure ventilation. ${ }^{5-6}$

The Division of Obstetric and Gynecologic Infectious Diseases in Philippine General Hospital (PGH) released a protocol for the management of pregnant COVID-19 patients, in line with the recommendation of PIDSOG. The general principle in creating a process flow for COVID-related procedure is minimizing exposure and maximizing resources.

The PGH released guidelines to provide information in order to prepare and guide the healthcare workers and personnel involved in the management of patients who have been deemed COVID-19 positive, suspect or probable who require urgent surgical management. Patients seeking consult at the $\mathrm{OB}$ Admitting Section (OBAS) will be assessed by an $\mathrm{OB}$ resident on duty and will be subsequently admitted based on initial evaluation. Emergent cases are cases with immediate threat to life of women or fetus; examples of which are fetal distress, placenta previa in hemorrhage and eclampsia. On the other hand, urgent cases are those cases with maternal or fetal compromise that is not immediately life-threatening, such as repeat CS, dystocia, malpresentation, chorioamnionitis, congenital anomaly, and HIV infection.
The OB Admitting section (OBAS) and Ward 15, located at the $1^{\text {st }}$ floor of the UP-PGH, were designated as red-zone or COVID areas. All personnel working within these areas were expected to follow proper protocol and wear appropriate PPE as prescribed by the hospital. Steps in donning were as follows: hand hygiene, shoe covers, inner gloves, protective overalls, N-95 mask, goggles, head cover, hood, face shield and outer gloves. A safety officer monitored every step of the donning procedure. This was time-consuming but was essential and very critical in prevention of transmission. All pregnant patients coming in the hospitals were triaged, examined and monitored in Ward 15. Those patients requiring surgical management were brought to a designated COVID operating rooms (OR) located at the third floor. These ORs and its equipment, including the anesthesia machines, were designated for use for COVID-19 patients for the duration of the pandemic. These changes caused additional steps and demands on the health care system including adjustment of doctors, nurses and staff, modification of duty schedules and additional infection control measures.

In 1989, the American College of Obstetricians and Gynecologists (ACOG) committee on professional standards delivery stated that hospitals with obstetric services should have the capability to start a CS within 30 minutes from the time that the decision is made to execute the procedure. In a more recent guideline, in 2011, the National Institute of Clinical Excellence (NICE) also suggested that the general performance of an obstetric unit should be measured by the DDI. In patients that require immediate obstetrical intervention, such as life-threatening cases for mother or fetus, CS should be started within 30 minutes from the decision making and 30 to 75 minutes for those patients with maternal and fetal compromise that is not essentially life-threatening. A delay of more than 75 minutes, especially in patients who require immediate intervention is associated with poor outcome. ${ }^{7}$

In a published practice guideline by the Royal College of Obstetricians and Gynaecologists, universal use of a nationally accepted classification of urgency of CS was encouraged. The four classification system included: Category 1 (emergent), immediate threat to life of woman or fetus; Category II (urgent), maternal or fetal compromise that is not immediately life-threatening; Category III (scheduled), needing early delivery but no maternal or fetal compromise; and Category IV (elective), at a time to suit the woman and maternity team. ${ }^{8}$

In a study by Gupta et al., a prospective 1-year study in a tertiary care hospital evaluated the DDI, factors affecting it and effects on maternal and neonatal outcome. Out of 453 emergency CS, mean DDI was $36.3 \pm 17.2$ minutes for patients requiring immediate intervention, and $38.1 \pm 17.7$ min for non-essentially life-threatening conditions. Only $42.4 \%$ of the emergency CS fulfilled the recommended 30-minute DDI while $57.6 \%$ had a DDI of more than 30 minutes. The identifiable reasons for the delay were as 
follows: delay in shifting the patient to operation theater (22.1\%), anesthesia factors (18.1\%), and lack of resources or manpower (16.1\%). Maternal complications were seen in $3.3 \%$ (15) patients with $0.7 \%$ (3) non-survivors having a DDI of $91.0 \pm 97.0$ minutes while survivors had a DDI of $36.8 \pm$ 15.7 minutes. There was no significant association between DDI and occurrence of neonatal complications. ${ }^{9}$

Similar findings were shown in a cross-sectional study by Tashfeen et al. with no significant differences in the perinatal outcomes of emergency cases with a DDI of $<30$ minutes and those with DDI of 31-60 minutes. However, a DDI of > 60 minutes was significantly associated with poor neonatal outcomes such as increased rates of special care baby unit admission and low APGAR scores. In this study, specific steps that caused the delays include obtaining consent for the procedure, securing an available operating theater and transporting the patients to the OR. ${ }^{10}$

Another study by Dimitrov et al. determined the time for the different steps of CS, from the point of patient's arrival at the OR, up to the last stitch in 82 elective and emergent CS cases. Results showed that preparation of anesthesia took 23 minutes, opening time of the uterus 37 seconds, delivery of the fetus 53 seconds, closure of the uterus 17 minutes and the entire CS procedure 44.3 mins, with a total mean of stay of the patient of 90 minutes. It also showed that elective CS took longer time than an emergent procedure and the delivery of the fetus was longer in repeat than in a primary CS. ${ }^{11}$

However, in times of pandemic, health services functions need to be altered to accommodate infection control measures. The Pandemic Influenza Expert Group created a guidance for pandemic influenza, published last 2009, to facilitate planning by the Health Service Executive in advance of the emergence of the next influenza pandemic. Consistent and correct execution of appropriate infection control precautions to restrict nosocomial transmission. ${ }^{12}$

Forrester et al. published a study on precautions for OR team members during the COVID-19 pandemic. A taskforce was assembled to develop a common algorithm for PPE use. In conjunction with infectious disease experts, guidelines were developed based on potential transmission, risk of exposure, and conservation of PPE. ${ }^{13}$

Among parturients, an expert review by Boelig et al. recommended respiratory precautions and PPE given the risk of asymptomatic carriers and transmission. The use of hand hygiene with alcohol-based rub after every patient contact was highly recommended and appropriate donning and doffing was a highly critical step in prevention of transmission of the disease. In general, all efforts should be made to limit the movement of women from one care area to another. A designated operating room should be use for suspected or confirmed COVID-19 patient. Appropriate planning, including simulation training, should be done regarding planned, urgent, and emergent cesarean delivery. ${ }^{14}$

This study aimed to provide information that will help guide the Department of Obstetrics and Gynecology in planning and developing effective strategies that may help in the OR processes. Results from this study may also serve as baseline information for future investigators who will be conducting studies on a similar topic.

\section{OBJECTIVES OF THE STUDY}

\section{General Objective}

This study aims to determine time and motion in the operating room in emergent, urgent and scheduled cesarean sections among pregnant COVID-19 patients.

\section{Specific Objectives}

1. To determine the decision-to-delivery interval (DDI) in emergent and urgent cesarean sections during the COVID-19 pandemic, by analysis of the following data

a. Pre-Induction time

b. Pre-Incision time

c. Opening time

2. To determine the overall operative time in emergent and urgent cesarean sections during the COVID-19 pandemic, by analysis of the following data
a. Pre-Induction time
b. Pre-Incision time
c. Opening time
d. Closing time

\section{METHODOLOGY}

\section{Research Design}

We used a retrospective descriptive study design. The study protocol was approved by the Ethics Review Board.

\section{Study Setting and Population}

The study population included all pregnant patients who were suspected, probable or confirmed COVID-19 who delivered by CS at the PGH from March 15-August 31, 2020. Excluded in the study were patients who delivered vaginally and undelivered patients who were managed medically.

\section{Data Collection and Analysis}

Data was collected from patient charts (doctors' orders, nursing notes and anesthesia OR records) and encoded using Microsoft Excel. The following data were collected: time of admission, time of decision of the obstetrician-gynecologist to do the cesarean section, time of arrival at the operating room, induction time, cutting time, time of delivery of the baby and time surgery ended.

This is a time and motion study and the monthly average of the following outcomes were calculated: pre-induction time, pre-incision time and opening time. Monthly average DDIs of emergent and urgent CS cases were calculated by adding the induction time, pre-incision time and opening time durations, while overall operating time was calculated by adding pre-incision time, opening time and closing time. 


\section{Operational Definition}

1. COVID-19 suspect - patients with mild, severe or critical symptoms who was not tested or awaiting COVID test results ${ }^{15}$

2. Probable COVID-19 - patients with mild, severe or symptoms with inconclusive, inadequate or no available testing for $\mathrm{COVID}^{15}$

3. Confirmed COVID-19 - patients with positive COVID-19 test results patients ${ }^{15}$

4. Emergent CS - cases with immediate threat to life of women or fetus ${ }^{8}$

5. Urgent CS - cases with maternal or fetal compromise which is not immediately life-threatening ${ }^{8}$

6. Scheduled CS - cases needing early delivery but no maternal or fetal compromise ${ }^{8}$

7. Decision time - time when the surgeon decides to deliver the patient abdominally

8. Induction time - time anesthesia induction starts

9. Pre-induction time - time between decision time and induction time

10. Incision time - the time the surgery starts when the first incision is made

11. Pre-incision time - time between induction time and incision time

12. Time of delivery - the time when baby is delivered

13. Opening time - time between incision time and time of delivery

14. Decision-to-delivery interval - the interval in hours or minutes from the time of decision of the obstetrician to carry out CS to the time of delivery of the baby.

15. Overall operating time - total time of surgery including induction time

\section{RESULTS AND DISCUSSION}

Out of 355 obstetric patients admitted at the Department of Obstetrics and Gynecology, 160 underwent CS, increasing from 5 in March 2020 to 64 in August 2020 (Figure 1).

There were 97 patients who were COVID-confirmed (60.6\%), 48 (30\%) who were COVID-suspects and 15 (9.4\%) who were COVID-probable patients upon admission (Table 1).

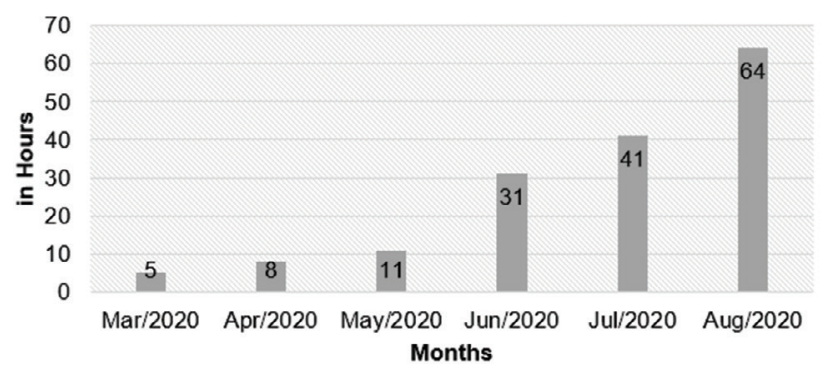

Figure 1. Distribution of patients who underwent CS at PGH per month.
The most common indications for abdominal delivery were as follows: repeat CS (79, 49.4\%), non-reassuring fetal status (NRFS) $(26,16.3 \%)$ relative maternal indication $(19,11.9 \%)$, and dystocia $(19,11.9 \%)$ (Table 2).

Repeat CS, relative maternal indication, dystocia, malpresentation, congenital anomaly, chorioamnionitis and HIV infection were considered urgent cases, while NRFS, placenta previa, and eclampsia were emergent. A total of 122 , (76\%) patients were urgent cases, 32 (20\%) were emergent, while 6 (4\%) were scheduled CS - 1 relative maternal indication, 4 repeat and 1 congenital anomaly (Table 3 ).

\section{Pre-Induction Time (Interval A)}

Pre-induction time coincided with activation of the OR team and included decision, confirmation of OR availability, huddle, OR preparation and transport.

The highest average pre-induction time was recorded in March 2020 ( 7 hours and 3 minutes). Pre-induction time decreased in the succeeding months to an average of 2 hours and 4 minutes for the last month of the study (Figure 2).

\section{Pre-Incision Time (Interval B)}

Pre-incision time included induction of anesthesia, prepping and draping. This outcome was relatively stable, seemingly not affected by the pandemic. The initial average time duration was 11 minutes in March 2020. It increased to 18 minutes (April) and ranged between 15.69 to 19.48 until August 2020 (Figure 3).

\section{Opening Time (Interval C)}

The average opening time in March was 15 minutes. It decreased to 9 minutes in April, but increased to 14 minutes

Table 1. COVID-19 classification

Patient's COVID Classification Number of Patients (\%)

$\begin{array}{ll}\text { Confirmed } & 97(60.6 \%) \\ \text { Suspect } & 48(30.0 \%) \\ \text { Probable } & 15(9.4 \%)\end{array}$

Table 2. Indications for abdominal delivery

\begin{tabular}{cc}
\hline Indications & Number of Patients (\%) \\
Repeat cesarean section & $79(49.4 \%)$ \\
Non-reassuring fetal status & $26(16.3 \%)$ \\
Relative maternal indication & $19(11.9 \%)$ \\
Dystocia & $19(11.9 \%)$ \\
Malpresentation & $7(4.4 \%)$ \\
Placenta previa & $4(2.5 \%)$ \\
Eclampsia & $2(1.3 \%)$ \\
Congenital anomaly & $2(1.3 \%)$ \\
Chorioamnionitis & $1(0.6 \%)$ \\
HIV infection & $1(0.6 \%)$ \\
\hline
\end{tabular}

Table 3. Classification of urgency of cesarean section

\begin{tabular}{cc}
\hline Classification & Number of Patients (\%) \\
Urgent & $122(76 \%)$ \\
Emergent & $32(20 \%)$ \\
Scheduled & $6(4 \%)$ \\
\hline
\end{tabular}


in May. It became stable in the last 3 months of the study with a range of 18 to 19 (Figure 4).

\section{Closing Time (Interval D)}

Closing time included closure of the uterus and the layers of the abdomen up to the skin closure. After an initial average closing time of 1.06 hrs. In March 2020, it decreased to $0.87 \mathrm{hrs}$. in April. For the remaining four months, it ranged from 1.21 to 1.38 hours (Figure 5).

\section{Decision-to-Delivery Interval (Interval A-C)}

No emergent cases were recorded during March 2020. The average 6 -month DDI on emergent cases $(n=32)$ was 2 hours and 38 minutes, while it was 3 hours and 51 minutes

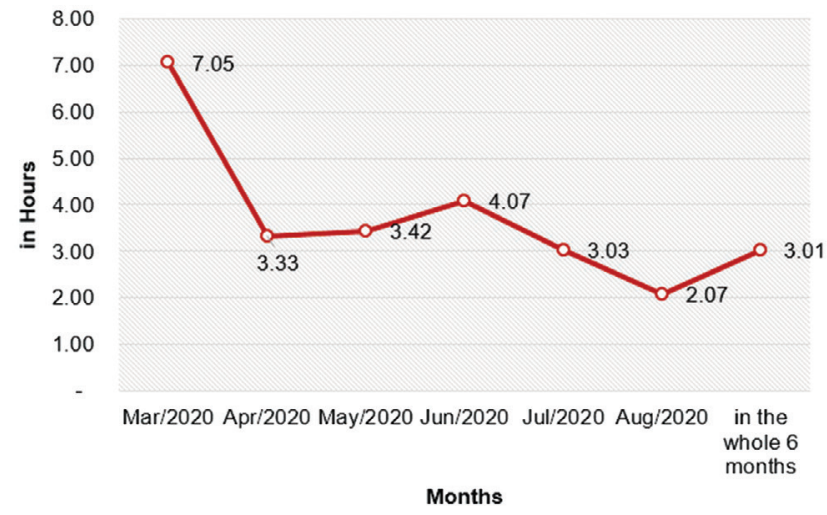

Figure 2. Average pre-induction time.

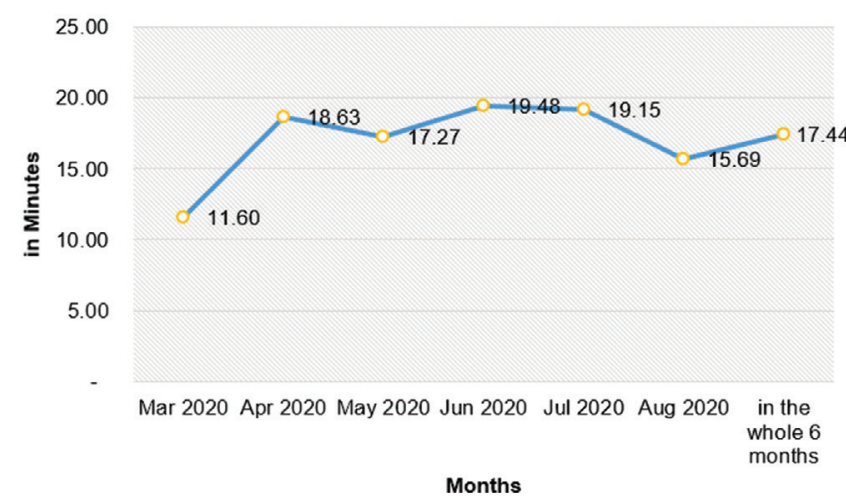

Figure 3. Average pre-incision time.

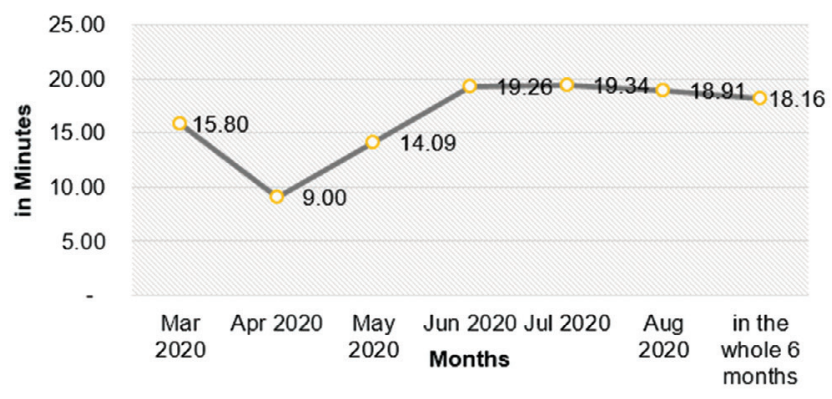

Figure 4. Average opening time. in urgent cases $(n=122)$. Results showed that the 30-minute DDI recommendation of the ACOG and NICE guidelines in both emergent and urgent cases were unattainable. The delays in DDI were caused by prolonged pre-induction time (Figure 6).

\section{Overall Operative Time (Interval B-D)}

The overall operative time monthly. No emergent cases were recorded during March. The average overall operative time for emergent cases during the 6-month study period was 1 hour and 31 minutes, and 1 hour and 57 minutes in urgent cases (Figure 7). The calculated overall operative time

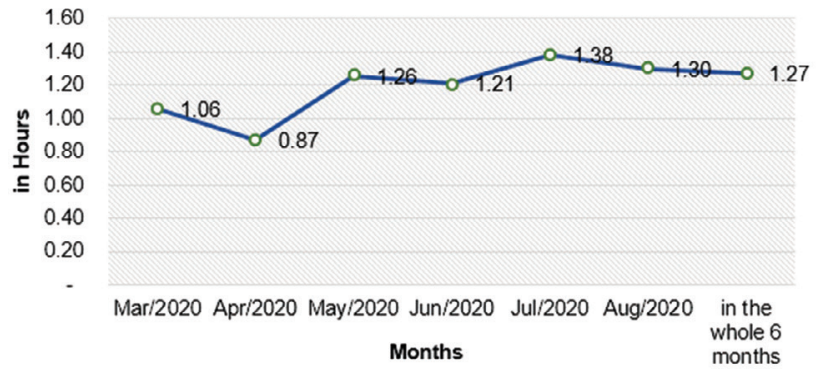

Figure 5. Average closing time.

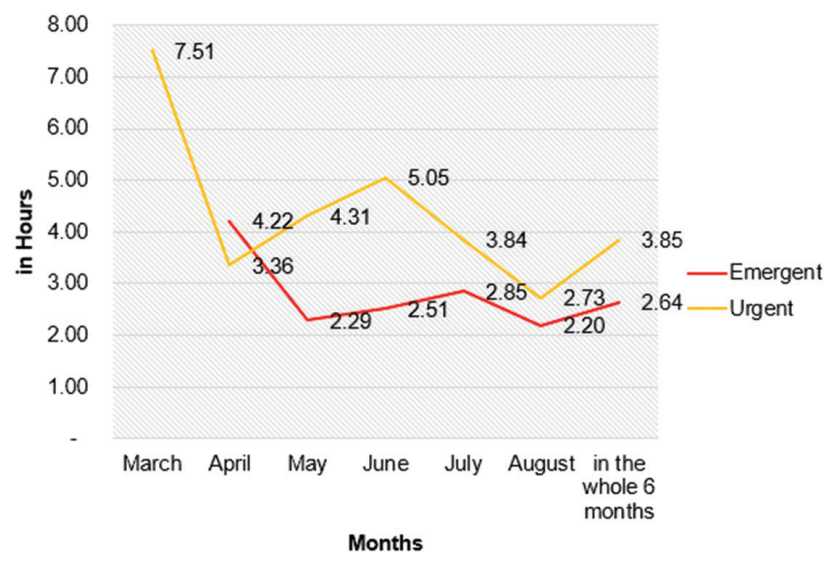

Figure 6. Decision-to-delivery interval (DDI).

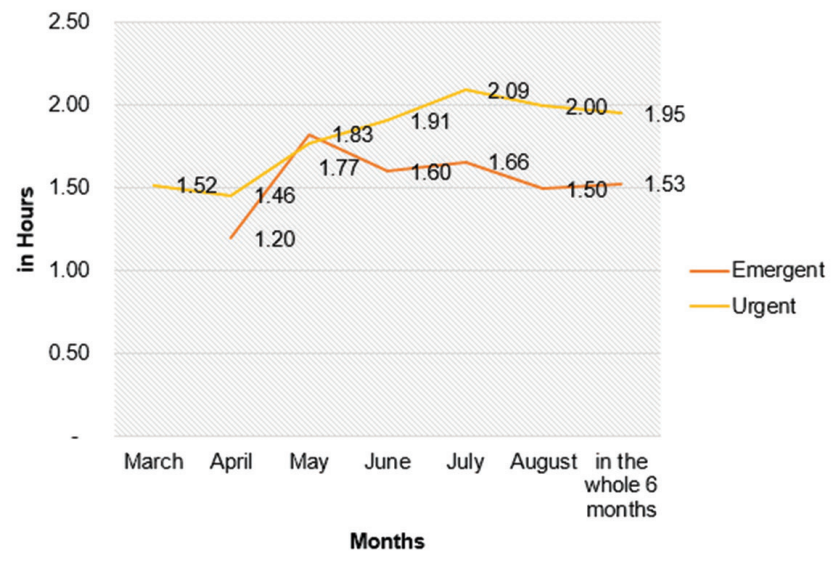

Figure 7. Average overall operating time. 
in both emergent and urgent cases were not in line with the average total CS time recommendation.

\section{DISCUSSION}

During this time of pandemic, the hospital created a new safety protocol to protect the patients and healthcare workers. These additional steps contributed to the delay of the delivery even in patients that needed emergent and urgent surgical intervention.

Emergent CS are cases with immediate threat to the life of the women or fetus, such as fetal distress, placenta previa in hemorrhage and eclampsia. These cases required immediate surgical intervention to prevent morbidity or mortality. On the other hand, urgent CS are cases with maternal or fetal compromise that are not immediately life threatening. Indications for CS in this study included repeat CS, relative maternal indications, dystocia, malpresentation, chorioamnionitis, congenital anomaly, and HIV infection. In the 6-month study, DDI (2 hours and 38 minutes) and overall operative time (1 hour and 31 minutes) for emergent cases were shorter than the DDI ( 3 hours and 51 minutes), and overall operative time (1 hour and 57 minutes) for urgent cases. Shorter and faster steps in the safety protocol were implemented in emergent cases. However, in both emergent and urgent cases, the recommended decision-to-delivery interval of 30 minutes (ACOG and NICE guidelines) and the average duration of 44.3 minutes for CS delivery were not feasible.

There were different reasons that caused the delay in each of the interval (A-C). In the pre-induction time (Interval A), during the preparation of the patient prior to CS, added protocol measures to prevent transmission of COVID and protect the patient and healthcare workers contributed to the delay. When the obstetrician-in-charge decided that a COVID patient needs surgery, he/she would coordinate with the anesthesiologist and $\mathrm{OR}$ nurse to activate the COVID OR team. The OR charge nurse would confirm the availability of an operating room, inform the anesthesia team and assign a scrub nurse, circulating nurse, nurse runner and attendant. A huddle would be called for a short briefing in order for the team to discuss OR needs and possible complications that may be encountered. A huddle was composed of the surgeons, anesthesiologists, pediatric catcher, OR nurses, circulating nurse and nurse runner. After the huddle, the circulating nurse and OR nurse would prepare the operating room, anesthesia pack, instruments and PPEs. Upon the signal of the OR charge nurse, the ward nurse would activate the ward transport team. The patient would be endorsed and transferred from the first floor to the COVIDOR located at the third floor. Factors that contributed to the delay were: additional time during huddle, prolonged preparation of the OR suite, availability of OR suite, delay in endorsement and preparation of patient, additional time required during donning, difficulty in transferring/clearing the path of the patient to the OR suite and shifting schedule of nurses. These factors were due to the reassignment of health care workers to deal with the pandemic for the first time. Results showed that DDI decreased from March to August 2020; the adjustments made by the hospital and health care workers contributed to this. In the beginning of the pandemic, simultaneous donning of all operative staff was time consuming and caused delay. However, in later months, donning was done per batch; OR nurses and anesthesiologists donned first, followed by the surgeons and lastly, the pediatric catcher.

During the pre-incision time (Interval B), Anesthesia factors, time in prepping and draping of the patient could have contributed to the delay. Although senior anesthesiologists were the ones handling the COVID-19 patients during this pandemic, there were still some technical factors that caused delay.

In the opening time (Interval C), obstetrician factors were responsible for extended time for the delivery. During the first three months of the study, only fellows-in-training were allowed to do CS. Fellows-in-training have already graduated from residency training and had more exposure to CS deliveries compared to the residents-in-training. However, since the study site was a training hospital, and to prepare for the possible influx of patients, they started to include residents in the procedure. The surgeon's skills may have also been part of the reasons for the long delivery of the baby. Additionally, complications such as adhesions due to previous surgery could also have been a factor.

Obstetrician factors also contributed to the delay in the closing time (Interval D). Uterine closure is a key step in CS to prevent future complications, thus an optimal surgical technique must be employed. Surgeon's skills may have caused prolonged closing time during CS, and thus also prolonged the overall operative time (Interval B-D).

Limitations of this study include the lack of documentation of the huddle time, length of OR preparation and transport of patient to the operating room, to be able to predict the cause of delay during pre-induction time. More in depth analysis of the surgical procedures and skills of the surgeon were also not documented. Analysis of maternal and neonatal outcomes were not included in the study.

The investigators would like to recommend 1) constant training and reinforcement of safety protocols for hospital staff, 2) monitoring and documentation of modifiable causes of delay to decrease the DDI time and overall operative time. 3) further study on the impact of the delay in correlation with maternal and neonatal outcomes.

\section{CONCLUSION}

The COVID-19 pandemic has negatively affected the provision of surgical obstetric care and OR utilization. Because of the new safety protocol for healthcare workers and patients, there was a significant delay from decision 
to delivery and overall operative time for both emergent and urgent cases. The possible causes of delay were either attributed to preparation factor (Interval A), anesthesia factor (Interval B) and obstetrician factor (Interval C to D). Identifying obstacles that are modifiable for the delay at different intervals may improve the decision-to-delivery interval, the overall operative time, and the quality of maternal and child birth care during this pandemic.

\section{Statement of Authorship}

All authors participated in the data collection and analysis and approved the final version submitted.

\section{Author Disclosure}

All authors declared no conflicts of interest.

\section{Funding Source}

This paper was self-funded.

\section{REFERENCES}

1. World Health Organization. (2020). Coronavirus disease (COVID-19) pandemic. [Internet]. [date cited]. Available from: World Health Organization: https://www.who.int/emergencies/diseases/novelcoronavirus-2019

2. Department of Health, Republic of the Philippines. (2020, April). UPDATES ON NOVEL CORONAVIRUS DISEASE (COVID-19). [Internet]. [date cited]. Available from: Department of Health: https://doh.gov.ph/2019-nCoV

3. Huang C, Wang Y, Li X, Ren L, Zhao J, Hu Y, et al. (2020). Clinical features of patients infected with 2019 novel coronavirus in Wuhan, China. Lancet (London, England), 395(10223), 497-506. https://doi. org/10.1016/S0140-6736(20)30183-5

4. Philippine Society for Microbiology and Infectious Diseases; Philippine College of Chest Physicians; Philippine College of Physicians; Philippine Rheumatology Association; Philippine College of Hematology and Transfusion Medicine. (2020, March 31). 4. Interim Guidelines on the Clinical Management of Adult Patients with Suspected or Confirmed COVID-19 Infection Version 2.1. Available from: https://www.psmid.org/wp-content/uploads/2020/07/ Final-PCP-PSMID-PCCP-COVID-19-Guidelines-March312020. pdf

5. Philippine Infectious Diseases Society for Obstetrics and Gynecolgy, Inc; Philippine Obstetrical and Gynecological Society (Foundation), Inc.; (2020, October). Retrieved from PIDSOG Handbook: A Guidance for Clinicians on the Obstetric Management of Patients with Coronavirus Disease 2019 (COVID-19), Second Edition;: Available from: https://pogsinc.org/wp-content/uploads/2020/11/ POGS-PIDSOG-COVID19-Handbook-2nd-edition-Oct-2020FINAL-COPY.pdf
6. Dashraath P, Wong J, Lim M, Lim LM, Li S, Biswas A, et al. (2020). Coronavirus disease 2019 (COVID-19) pandemic and pregnancy. American journal of obstetrics and gynecology, 222(6), 521-31. https://doi.org/10.1016/j.ajog.2020.03.021

7. National Institute for Health and Clinical Excellence. (2011, November 23). Caesarean section. Retrieved from https://www.nice. org.uk/guidance/cg132

8. Royal College of Obstetrician and Gynaecologist. (2010, April). CLASSIFICATION OF URGENCY OF CAESAREAN SECTION - A Continuum of Risk. [Internet]. [date cited]. Available from: Royal College of Obstetrician and Gynaecologist: https://www.rcog.org.uk/globalassets/documents/guidelines/ goodpractice11classificationofurgency.pdf

9. Gupta S, Naithani U, Madhanmohan C. Singh A, Reddy P, Gupta A. (2017). Evaluation of decision-to-delivery interval in emergency cesarean section: A 1-year prospective audit in a tertiary care hospital. Journal of anaesthesiology, clinical pharmacology, 33(1), 64-70. https://doi.org/10.4103/0970-9185.202197

10. Tashfeen K, Patel M, Hamdi IM, Al-Busaidi I, Al-Yarubi N. (2017). Decision-to-Delivery Time Intervals in Emergency Caesarean Section Cases: Repeated cross-sectional study from Oman. Sultan Qaboos University medical journal, 17(1), e38-e42. https://doi.org/10.18295/ squmj.2016.17.01.008

11. Dimitrov A, Stamenov G, Krŭsteva K. (1999). Obshta i poetapna prodŭlzhitelnost na tsezarovoto sechenie [The overall and step-by-step duration of cesarean section]. Akusherstvo i ginekologiia, 38(3), 7-10.

12. Pandemic Flu Team, Department of Health, England Health Protection Agency. (2009, December). [Internet]. [date cited]. Available from: PANDEMIC (H1N1) 2009 INFLUENZA. A summary of guidance for infection control in healthcare settings: https://assets. publishing.service.gov.uk/government/uploads/system/uploads/ attachment_data/file/361997/Pandemic_influenza_guidance_for_ infection_control_in_critical_care.pdf

13. Forrester JD, Nassar AK, Maggio PM, Hawn MT. (2020). Precautions for Operating Room Team Members During the COVID-19 Pandemic. Journal of the American College of Surgeons, 230(6), 1098-1101. https://doi.org/10.1016/j.jamcollsurg.2020.03.030

14. Boelig RC, Manuck T, Oliver E.A, Di Mascio D, Saccone G., Bellussi F, Berghella V. (2020). Labor and delivery guidance for COVID-19. American journal of obstetrics \& gynecology MFM, 2(2), 100110. https://doi.org/10.1016/j.ajogmf.2020.100110

15. Department of Health, Republic of the Philippines. (2020, April). INTERIM GUIDELINES ON THE COVID-19 DISEASE SEVERITY CLASSIFICATION AND MANAGEMENT. [Internet]. [date cited]. Available from: Department of Health: https://doh.gov.ph/node/24520 